\title{
ALTERACIONES Y ALTERIDADES DEL ESPACIO EN LOS CUENTOS DE FELISBERTO HERNÁNDEZ Y HORACIO QUIROGA: UNA GEOPOÉTICA DE LO FANTÁSTICO
}

\author{
Audrey Louyer DaVo \\ Université de Reims Champagne-Ardennes \\ audrey.louyer@orange.fr
}

Recibido: 13-02-2013

Aceptado: 10-04-2013

(c) (1) $\Theta$

RESUMEN

Este trabajo contempla la relación entre el espacio físico y lo fantástico según tres ejes principales: el eje referencial, narrativo y de la recepción. Los aplicamos al estudio de varios cuentos de Horacio Quiroga y Felisberto Hernández para explicar la impresión de lo fantástico que sugieren sus textos a través de una concepción de lo fantástico como paso o pasaje. El espacio se revela así ser el marco de lo posible, el lugar alterado por la escritura y el campo de confrontación entre diferentes concepciones de los mundos posibles. Desarrollamos, pues, un concepto de "geopoética de lo fantástico", que analiza estos aspectos que surgen de la escritura de nuestros autores a partir de una perspectiva europea.

PALABRAS ClAVE: Quiroga, Hernández, pasaje, alteración, geopoética, fantástico

\section{AbSTRACT}

This work considers the relationship between physical space and fantastic literature through three main perspectives: setting, narrative techniques and reception. They are applied to the study of several of Horacio Quiroga's and Felisberto Hernández's short stories to explain the effect of the fantastic suggested by their texts, considering it as a passage. This way, space can be the scene where the action takes place in the framework of a possible world, a place altered by the way of writing, or an area of confrontation between different conceptions of possible worlds. We develop, from a European point of view, a concept of the "geopoetic of the fantastic", which analyzes these aspects that arise from these authors' way of writing.

KEYwORDS: Quiroga, Hernández, passage, alteration, geopoetic, fantastic 
Hay algo paradójico en la idea de examinar el espacio cuando el objeto de estudio, el cuento fantástico, es una creación hecha de palabras y no un objeto materialmente visual como lo sería un cuadro, una foto o una película. Si es verdad que en cierta época el análisis del tiempo tuvo su momento de gloria, que se ilustró con los conceptos desarrollados por los estructuralistas, verdad es que su correlativo, el espacio, se ha quedado al margen hasta el llamado spatial turn de los años ochenta. Y este espacio es sumamente importante a la hora de reflexionar sobre lo fantástico, ya que se suele definir este último como una dimensión nueva creada a partir del marco de lo real, o mejor dicho de lo posible, y que se inscribe en detalles, imágenes o lugares reconocibles e identificables por el lector. Los dos autores elegidos para nuestro estudio tienen como puntos comunes su nacionalidad, la época en que escribieron (primera mitad del siglo XX) y que ambos plantean el problema de ser reconocidos o no como autores que cultivan lo fantástico. ${ }^{1}$ Nuestro objetivo en este artículo será analizar las posibles relaciones que se establecen entre ambos y confirmar, a partir de un análisis del espacio y de su relación con lo fantástico, la pertenencia a dicho género de varios de sus cuentos. ${ }^{2}$

Antes de ir más lejos, es imprescindible precisar qué definición de lo fantástico vamos a utilizar a lo largo de este texto: en efecto, por ser numerosas las concepciones de lo fantástico, según las épocas, las áreas geográficas, las corrientes o tendencias analíticas, hay que delimitar los términos que utilizamos para hablar de lo fantástico. La nuestra radica en la teoría de Roas (2011) y viene completada por Muñoz Rengel (2009): se trata de concebir lo fantástico como una brecha en nuestro paradigma de realidad, a través de la sintaxis o la metaficción, que conduce a un miedo metafísico o intelectual, y cuestiona nuestra percepción de lo cotidiano; y menos que el tema propiamente dicho, lo fantástico estriba en la manera de tratarlo. Añadiremos que esta brecha nos parece fundamental cuando hablamos de «efecto fantástico»,

1 En el caso de Felisberto Hernández, por ejemplo, Italo Calvino y Julio Cortázar mantienen posiciones encontradas acerca de esta cuestión. Por una parte, afirma Cortázar que «La calificación de "literatura fantástica" me ha parecido siempre falsa, incluso un poco perdonavidas en estos tiempos latinoamericanos en que sectores avanzados de lectura y de crítica exigen más y más realismo combativo. Releyendo a Felisberto he llegado al punto máximo de este rechazo de la etiqueta "fantásti-

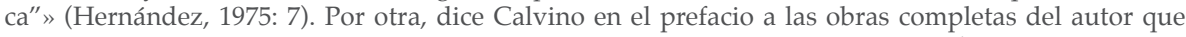
éste «llegó a conquistar un lugar propio entre los cultores del "cuento fantástico" hispanoamericano» (Hernández 1997: 25-26). Y por lo que se refiere a Quiroga, muchos de sus cuentos forman parte de lo maravilloso o se refieren al mundo infantil, de ahí la necesidad de mencionar los textos elegidos. 2 Esencialmente en «Las Hortensias», «La casa inundada», «El balcón», «El acomodador», «Tierras de la memoria», «Por los tiempos de Clemente Colling », «La pelota», «Muebles "El Canario"» y «Las dos historias», en el caso de Felisberto Hernández, en «El vampiro», «El espectro», «El hijo», «El almohadón de pluma», «A la deriva», «El síncope blanco», «El solitario» $\mathrm{y}$ « Los buques suicidantes», por lo que se refiere a Horacio Quiroga. 
ya que dicha brecha se podría interpretar como un pasaje hacia «otra cosa», a una realidad imposible pero que existe en el tejido de palabras y fascina al lector.

Los ejes de estudio para la relación que une el espacio y lo fantástico a partir de esta definición implican tener en cuenta el contexto de escritura, de recepción, la herencia cultural de los autores. Suponen también recordar que somos lectores europeos de una realidad hispanoamericana diferente de nuestra realidad, de ahí la idea de alteridad. En la medida en que nuestro estudio se centrará en la acepción física del espacio, también nos parece conveniente llevar a cabo nuestro trabajo utilizando instrumentos de la geografía, sobre todo a partir de diversos conceptos estudiados por Collot (2011) y Baron (2011).

Llegamos a la pregunta siguiente: ¿en qué medida un estudio geofantástico de una selección de cuentos permite revelar sus rasgos fantásticos, convirtiéndolos en taller de invención de formas alteradas del espacio? Así será nuestro viaje por las obras de Quiroga y Hernández: si, en un primer momento, el espacio es en muchos casos un escenario verosímil y posible que constituye el contexto de realización del efecto fantástico, limitarse a un mero marco sería reductor. En efecto, el espacio puede ser un actor de lo fantástico, en una dinámica de transgresión: las alteraciones de lo posible. Ahora bien, tratar el aspecto físico del espacio tampoco es sinónimo de reducir el estudio a una descripción, por lo que finalmente nos preguntaremos: ¿Dónde está lo fantástico? ¿Cómo pensar la alteridad gracias al espacio?

\section{ESPACIO-ESCENA: EL MUNDO OBSERVADO}

Veamos primero cómo aparece el espacio nombrado en nuestros textos y cuál es su papel: intentemos una cartografía de lo fantástico, empezando por el espacio-marco y terminando por el espacio construido.

\subsection{El espacio, marco de verosimilitud}

El espacio vivido por el autor aparece transcrito en su obra para crear un «con-texto», una escenificación de la acción en la que lo fantástico puede inmiscuirse para crear el sentimiento de lo ominoso. Según advierte Muñoz Rengel (2009: 11), «El relato fantástico busca ante todo la verosimilitud, construir un escenario lo más parecido posible a nuestra habitual interpretación del mundo, porque sólo en ese universo de leyes naturales podrá cuestionar el alcance y la validez de éstas». El papel del espacio consiste aquí en crear un contexto plausible que el lector se crea. 
En los textos de Quiroga, la toponimia contribuye a este efecto. Una región como Misiones, ciudades como Resistencia o la mención del río Paraná, sugieren lugares reales en los cuentos «A la deriva», «El alambre de púa» o «Yaguaí». Pero a veces, los lugares no necesitan ser nombrados para crear una impresión de autenticidad y constituir un marco espacial de referencia para el lector. En el caso de Felisberto Hernández, y a menudo ya en el título del cuento, vienen evocados los espacios citadinos y familiares, como el balcón, la casa (aunque inundada...), la habitación de un hotel, el tranvía, y éstos dan una impresión de lo cotidiano que permite al lector representarse el espacio para apropiárselo mejor, generándose así un ambiente conocido antes de crear el desfase con lo fantástico.

La casi totalidad de los relatos de Felisberto Hernández suceden en la ciudad. Es el lugar de lo moderno, de la novedad, de la sorpresa, pero también un espacio ordenado y ocupado por el hombre, y por eso mismo puede cobrar una dimensión fantástica. A veces se trata de Montevideo, que aparece abiertamente nombrada, o que adivinamos a través de unos nombres de calles, remitiendo, así, a un referente reconocible o imaginable. Por ejemplo, al final del cuento «Las Hortensias», el protagonista deambula por la ciudad y va adonde le llevan sus pensamientos hasta encontrarse como por casualidad en un parque: «despertó de sus pensamientos en el Parque de las Acacias y fue a sentarse en un banco. Mientras pensaba en su vida dejó la mirada debajo de unos árboles y después siguió la sombra, que se arrastraba hasta llegar a las aguas de un lago» (Hernández, 2008: vol. 2, 226). Efectivamente -y gracias al acceso a los mapas precisos en Internet- nos enteramos de que existe un parque con este nombre en la capital uruguaya.

\subsection{El marco de lo posible: el espacio construido}

Sin embargo, por más realista que parezca este marco, se trata de un espacio necesariamente construido por el texto. De hecho, las descripciones de marcos posibles se parecen al efecto de lo real explicado por Roland Barthes (1982: 89):

c'est là ce qu'on pourrait appeler l'illusion référentielle. La vérité de cette illusion est celle-ci : supprimé de l'énonciation réaliste à titre de signifié de dénotation, le «réel» y revient à titre de signifié de connotation; car dans le moment même où ces détails sont réputés dénoter directement le réel, ils ne font rien d'autre, sans le dire, que le signifier [...] Il se produit un effet de réel, fondement de ce vraisemblable inavoué qui forme l'esthétique de toutes les œuvres courantes de la modernité. 
Tomemos por ejemplo el cuento «El acomodador»: se instaura un ambiente fantástico en el que el autor inserta elementos que crean un efecto de ilusión referencial: «Una noche me desperté en el silencio oscuro de mi pieza y vi, en la pared empapelada de flores violetas, una luz. Desde el primer instante tuve la idea de que me ocurría algo extraño, y no me asusté [...] Bajé los ojos hasta la mesa y vi las botellas y los objetos míos. No me quedaba la menor duda; aquella luz salía de mis propios ojos» (Hernández, 2008: vol. 2, 78-79). El papel pintado de la pared así como la mención de la mesa y las botellas son motivos espaciales que permiten insertarse en un mundo posible, y al final de la frase se puede producir el paso a lo increíble.

Ahora bien: nos parece que hay que distinguir el espacio referencial y el espacio imaginado. Cuando el autor elige unos rasgos específicos de un espacio para crear un efecto particular, se trata de una «re-creación» del espacio que supone una apropiación. Así lo confirma Gilbert Durand (1992: 473), apoyándose en Sartre y Piaget: «L'espace alors devient superlatif et quitte le domaine de l'indifférente "localisation" pour engager l'image dans "l'appartenance". Cette distinction d'un "espace perceptif" et d'un "espace représentatif" est également la conclusion de l'étude très serrée de Piaget». En resumidas cuentas, opone la mera localización a la pertenencia y, como consecuencia, el espacio de la percepción y el de la representación. Es lo que pasa por ejemplo en «Las hortensias»: «Aquella habitación sería un presidio en un castillo, el piano hacía ruido de tormenta y en la ventana aparecía, a intervalos, un resplandor de relámpagos; entonces recordó que hacía unos instantes las ruedas de la tarima le hicieron pensar en un trueno lejano; y esa coincidencia lo inquietó» (Hernández, 2008: vol. 2, 183). Se trata aquí de una creación de un espacio hostil con una selección precisa de los detalles visuales y auditivos que son el castillo, el trueno, los chillidos y el piano (elementos en los que, quizá, pueda leerse una intención paródica de los motivos clásicos de lo fantástico).

Cuando no es el marco de la acción, el espacio puede constituir el escenario de las emociones de los personajes, su «mundo interior». Lo fantástico, en su dimensión de paso, estriba entonces en la manera cómo el narrador conduce al lector por sus pensamientos como nos llevaría un guía por un espacio desconocido: se trata de descubrir, a través de la escritura, un mundo interior estructurado de análogo modo al espacio físico y exterior. Este procedimiento se observa a menudo en Felisberto Hernández: «A veces mis pensamientos están reunidos en algún lugar de mi cabeza y deliberan a puertas cerradas: es entonces cuando se olvidan del cuerpo» («Tierras de la memoria»; Hernández, 
2008: vol. 3, 33). Del mismo modo, la personificación de los pensamientos, la percepción y la interpretación de los acontecimientos exteriores contribuyen al efecto fantástico del mundo interior de los personajes. Se trata de algo similar a lo que Bertrand Westphal denomina «politopía», y define como «l'espace secret, l'espace de l'hyperbate, celui où l'individu déploie un supplément de vérité personnelle à l'abri des yeux du monde, des prescriptions du code» (Westphal, 2007: 75). Si pensamos en esta idea a partir de la concepción de lo fantástico según Todorov (1970), podemos pensar que se trata nada más de la tradicional vacilación entre una explicación racional, científica, que sería el espacio de lo objetivo, y la explicación personal y subjetiva del personaje, loco o lúcido, y cuyo mejor ejemplo puede ser el narrador de «Le Horla» de Maupassant. Pero nos parece que nuestros autores dan otro matiz a esta idea. En el caso de Horacio Quiroga, observemos cómo se registran diversas percepciones del espacio en el cuento «A la deriva»: se trata de la lenta agonía de un hombre mordido por una yarará que intenta alcanzar un pueblo navegando por el Paraná, lo que ya prefigura simbológicamente el río Estigia y la barca de Caronte. La visión exterior de los ojos del mundo la da el narrador extradiegético: «Pero el hombre no quería morir, y descendiendo hasta la costa subió a su canoa. Sentóse en la popa y comenzó a palear hasta en centro del Paraná. Allí la corriente del río, que en las inmediaciones del Iguazú corre seis millas, lo llevaría antes de cinco horas a Tacurú-Pucú» (Quiroga, 1993: 53). Poco después, ya es otra la descripción del espacio: «El Paraná corre allí en el fondo de una inmensa hoya, cuyas paredes, altas de cien metros, encajonan fúnebremente el río» (Quiroga, 1993: 54). Y cuando el veneno que va difundiéndose por el cuerpo del personaje lo lleva a la insensibilidad, también se produce una transformación del espacio: «Y de pronto, con asombro, enderezó pesadamente la cabeza: se sentía mejor. [...] Calculó que antes de tres horas estaría en Tacurú-Pucú. El bienestar avanzaba y con él una somnolencia llena de recuerdos» (Quiroga, 1993: 54). A partir de este punto pasamos al desarrollo del espacio subjetivo del personaje.

Felisberto Hernández va más allá, y esto se revela a través de un fragmento como: «Yo creo que en todo el cuerpo habitan pensamientos, aunque no todos vayan a la cabeza y se vistan de palabras. Yo sé que por el cuerpo andan pensamientos descalzos» («Tierras de la memoria»; Hernández, 2008: vol. 3, 33). La elección del verbo «habitar» no es inocente y connota el aspecto fantasmagórico de la imagen. Pero además, los «pensamientos descalzos» son las ideas personales indecibles y no sometidas a los códigos sociales del 
espacio exterior. Se trata de una transgresión del mundo posible que crea una especie de polifonía de los espacios.

En estos ejemplos se hace evidente que el espacio mental es estructurado, construido y transmitido mediante una cartografía interior, en una politopía que va progresivamente de lo exterior a lo interior en el caso de Quiroga, mientras que en Felisberto Hernández se manifiesta a través de la introspección llevada a cabo en estas pausas en la narración. Estos elementos generan un efecto fantástico al constituir un pasaje que da al lector la visión de una vida independiente de su conciencia.

\subsection{Los limites del espacio}

Utilizar el espacio físico como marco tranquilizador es un artificio eficaz de los escritores fantásticos, aunque cuestionable para todo lector consciente de los mecanismos de la escritura. En efecto, el pretendido realismo de los mapas y de la topografía no es más que una ilusión, como ya lo decía Borges al llevar al extremo absurdo la supuesta objetividad de los mapas en su microrrelato «Del rigor de la ciencia»:

En aquel imperio, el arte de la cartografía logró tal perfección que el mapa de una sola provincia ocupaba toda una ciudad, y el mapa del imperio, toda una provincia. Con el tiempo, estos mapas desmesurados no satisficieron y los colegios de cartógrafos levantaron un mapa del imperio, que tenía el tamaño del imperio y coincidía puntualmente con él. Menos adictas al estudio de la cartografía, las generaciones siguientes entendieron que ese dilatado mapa era inútil y no sin impiedad lo entregaron a las inclemencias del sol y los inviernos. En los desiertos del oeste perduran despedazadas ruinas del mapa, habitadas por animales y por mendigos; en todo el país no hay otra reliquia de las disciplinas geográficas (Borges, 1989: 847).

En este cuento, la geografía se ha vuelto una ciencia inútil porque ha borrado la frontera entre lo que percibimos y la representación de esta percepción, como si los dos mundos pudieran superponerse. El choque entre ambos revela la inutilidad de esa extrema exactitud y termina provocando, gracias a la habilidad de Borges, un inquietante efecto fantástico.

No hay que olvidar que un espacio objetivo no existe, sino que es el producto de una doble interpretación, la del autor y la del lector. Así lo afirma Collot (2011): «il n'existe nulle coïncidence entre le plan d'une ville dont nous consultons le dépliant et l'image mentale qui surgit en nous, à l'appel de son nom, du sédiment posé dans la mémoire par nos vagabondages quotidiens». 
Por eso, en nuestros autores, la pretendida objetividad del espacio-marco se tiene finalmente que matizar: a pesar de ser la imagen de un mundo posible, es necesariamente un espacio creado por la escritura. Veamos entonces cómo el texto juega con el espacio para que surja lo fantástico, en términos de creación de brechas. Resulta imposible hacer una lista detallada de todos los recursos - tampoco nos parece pertinente ofrecer aquí un catálogo-, pero el análisis de los motivos, objetos y técnicas de la interacción entre el espacio y lo fantástico resultará de gran ayuda para el estudio de dicho género.

\section{ESPACIO-ACTOR: VECTORES DE ALTERACIÓN}

En términos científicos, un vector es un segmento de recta caracterizado por un origen, un módulo (longitud), una dirección (ángulo) y un sentido (indicado con una flecha). Permite la migración de una figura hacia otro lugar del espacio determinado. En los cuentos fantásticos, la realidad expuesta se traslada como si ocurriera bajo la influencia de vectores para pasar a otra cosa, a otro mundo. Veamos cómo se realiza.

\subsection{Motivos visuales}

Las sombras constituyen un recurso temático frecuente de lo fantástico. Una proyección de un objeto o un ser que puede volverse fantástica, por ejemplo, a partir del momento en que adquiere una autonomía independientemente de su poseedor. Eso implica también una alteración del espacio: «no sólo los objetos tenían detrás una sombra, sino que también los hechos, los sentimientos y las ideas tenían una sombra. Y nunca se sabía bien cuándo aparecía ni dónde se colocaba. Pero si pensaba que la sombra era una seña de misterio, después me encontraba con que el misterio y su sombra andaban perdidos, distraídos, indiferentes, sin intenciones que los unieran» («Por los tiempos de Clemente Colling»; Hernández, 2008: vol. 1, 198). Además de las alegorías de las ideas que cobran una espesura textual y ocupan el espacio de manera sorprendente, este fragmento nos recuerda otra alegoría, la de la caverna de Platón. En ésta, el personaje ve cómo se mueven de manera extraña unas figuras por una pared, hasta que comprende - al cambiar su punto de visión-que las figuras son las sombras proyectadas de unos esclavos encadenados e iluminados por un farol. Un movimiento hacia el conocimiento y la verdad. Esta imagen podría ser una explicación de lo que es lo fantásti$\mathrm{co}$, que se corresponde con la conciencia de la existencia de otro paradigma: las figuras que considerábamos como seres autónomos no eran más que una 
proyección que interpretamos a partir de nuestra percepción limitada. Saber percibir a los esclavos es ver otro mundo posible, quizás en mayor adecuación con el conjunto de la verdad ya que incluye dentro de él, de manera coherente, el primer mundo, el mundo de las figuras, inexplicable a primera vista. El interés del texto de Platón consiste en ayudarnos a salir de la caverna para alcanzar la deslumbrante verdad.

Directamente vinculado con esta idea, está el cine, un arte que tiene una presencia recurrente en los cuentos de Quiroga como elemento creador de efectos fantásticos. Acostumbrado al teatro, Quiroga opera una confusión entre dos espacios: el del rodaje con los actores y el de la pantalla (que refleja la imagen del proyector). Así, en «El vampiro» imagina que los personajes proyectados en la pantalla tienen una vida independientemente de la película y de los mismos actores. En esta confusión se produce la brecha que crea el efecto fantástico. Pero Quiroga va más lejos con este juego metaficcional en su cuento «El espectro», donde borra las fronteras entre la obra de ficción y el marco espacial de su realización al convertir la propia sala de cine en el lugar del duelo entre dos hombres rivales de un triángulo amoroso, siendo uno de ellos un miembro del público y el otro el héroe de la película.

Pero a despecho de las leyes y los principios, Wyoming nos estaba viendo. Si para la sala El Páramo era una ficción novelesca, y Wyoming vivía sólo por una ironía de la luz; si no era más que un frente eléctrico de lámina sin costados ni fondo, para nosotros - Wyoming, Enis y yo- la escena filmada vivía flagrante, pero no en la pantalla, sino en un palco, donde nuestro amor sin culpa se transformaba en monstruosa infidelidad ante el marido vivo. [...] Sentí que la piel de la espalda se me erizaba, y miré: con lentitud de fiera y los ojos clavados en nosotros, Wyoming se incorporaba del diván. Enid y yo lo vimos levantarse, avanzar hacia nosotros desde el fondo de la escena, llegar al monstruoso primer plano... Un fulgor deslumbrante nos cegó, al tiempo que Enid lanzaba un grito. La cinta acababa de quemarse (Quiroga, 1993: 548-549).

Este fragmento permite ilustrar la confusión de los dos mundos. Y nos parece que la antepenúltima frase ilustra también el concepto de «frase umbral» desarrollado por Patricia García (2013). Este concepto se refiere a un fenómeno presente en ciertos cuentos en los que una frase constituye el empalme, el cruce entre dos paradigmas, basado en una ambigüedad referencial gracias a recursos lingüísticos. Se trata de ver lo fantástico como un desliz y no como una irrupción, según la célebre definición de Caillois. ${ }^{3}$ En nuestro

3 «La démarche essentielle du fantastique est l'Apparition: ce qui ne peut pas arriver et qui se pro- 
caso pues, el público presente para ver la película se reduce a los dos protagonistas, como un efecto de zoom. Luego, la referencia de lugar «desde el fondo de la escena» crea una ambigüedad ya que puede ser la escena de la película pero también la escena del teatro donde se proyecta. Por último, cuando parece que el personaje cruza la tela de la pantalla, el fulgor es la manifestación de que ocurrió lo imposible.

\subsection{Objetos y lugares de alteración}

El espejo es el primer elemento que vamos a analizar. Basta pensar en la fotografía de Chema Madoz que representa una escalera apoyada contra un espejo, creando de esta forma una ilusión de óptica como si el espectador pudiera pasar «al otro lado del espejo» y bajar a otro mundo: en esta imagen se hace evidente toda la simbología que solemos asociar a este objeto mágico y angustiante a la vez. Es importante destacar que en francés espejo es «miroir», palabra que deriva del latín mirari, que significa «asombrarse». Examinado desde el punto de vista del espacio, el espejo se convierte en un objeto muy sugerente, en la medida en que al reflejar una imagen es capaz de crear una sensación de vértigo, de inversión y de paso hacia «otra cosa». No es casual la elección del título de Le miroir de sorcière por parte de Jean Fabre (1992) para su estudio sobre lo fantástico.

Resulta revelador que Pigmalión, personaje del cuento «Las Hortensias» de Felisberto Hernández, no soporte contemplar su propio reflejo: «Al pasar frente a un espejo, y a pesar de estar corrida la cortina, vio a través de ella su cara: algunos rayos de sol daban sobre el espejo y habían hecho brillar sus facciones como las de un espectro. Tuvo un escalofrío, cerró las ventanas y se acostó» (Hernández 2008: vol. 2, 210). Una acción que subraya el malestar que sentimos al ver de otra manera el mundo conocido y al descubrir cómo nos ve otra persona, a través de una impresión de enajenación y de desdoblamiento. El miedo de Pigmalión le hace plantearse «que podrían inventar espejos en los cuales se vieran los objetos pero no las personas», (Hernández, 2008: vol. 2, 212). Una imagen que evidencia el miedo metafísico que define a lo fantástico (Roas, 2011: 94-107): si el espejo nos ofrece un reflejo de la realidad, no aparecer en él nos convierte en nada, en un sinsentido.

A veces, un mero acto cotidiano puede convertirse en fantástico, gracias a la habilidad del narrador: «El espejo [...] era bajo y Horacio podía pa-

duit pourtant, en un point et à un instant précis, au cœur d'un univers parfaitement repéré et d'où l'on avait à tort estimé le mystère à jamais banni» (Caillois, 2008: 680-681). 
sar, distraído, frente a él e inclinarse, todos los días, hasta ver solamente el nudo de la corbata; se peinaba de memoria y se afeitaba tanteándose la cara. Aquel espejo podía decir que él había reflejado siempre un hombre sin cabeza», (Hernández, 2008: vol. 2, 209). En este caso, mediante la personificación del espejo pero sobre todo con el final que nos da la impresión de un hombre degollado, es la escritura la que altera la visión. Es un acto bastante trivial, pero que se vuelve fantástico con la técnica de la deformación.

Otro de los elementos esenciales para la creación del efecto fantástico en la obra de Horacio Quiroga es la selva. En su cuento «El hijo», en el que un padre busca en vano a su hijo que se fue por la selva, este espacio se presenta como un lugar hostil a la vida humana. Ya desde el inicio, la selva aparece descrita en esos términos negativos: «El sol, ya muy alto, continúa ascendiendo. Adonde quiera que se mire — piedras, tierra, árboles_-, el aire, enrarecido como en un horno, vibra con el calor. Un profundo zumbido que llena el ser entero e impregna el ámbito hasta donde la vista alcanza, concentra a esa hora toda la vida tropical», (Quiroga, 1993: 754). Mientras el padre se mantiene fuera de la selva, está casi persuadido de que su hijo ha muerto. El paso hacia lo fantástico se opera cuando sale a buscarlo, lo encuentra mágicamente en la selva y vuelve a casa con él. Sólo el hábil cambio de perspectiva al final del cuento (el narrador pasa a ser omnisciente) revela el delirio del personaje: «Sonríe de alucinada felicidad... Pues ese padre va solo. A nadie ha encontrado, y su brazo se apoya en el vacío», (Quiroga, 1993: 756).

La hostilidad de la selva también se observa en otros dos cuentos, «A la deriva», del que ya hemos hablado, y «La miel silvestre», cuento en el que el goloso protagonista queda paralizado por haberse comido la miel que les robó a las abejas, y termina castigado y devorado por las hormigas carnívoras. Si en el caso de Felisberto, se trata de utilizar un objeto de lo cotidiano para provocar el traslado, es más bien todo un espacio que le resulta hostil al hombre que viene presentado y descrito en los cuentos de Quiroga.

\subsection{Técnicas del texto}

La subjetividad del recuerdo es el primer aspecto que vamos a desarrollar. En Felisberto Hernández, la descripción de unos personajes que el protagonista conoció tiempo atrás, sobre todo cuando se refieren a un pasado lejano, puede resultar bastante extraña. Si bien es cierto que la memoria intenta restituir una experiencia a partir de los recuerdos, a menudo las descripciones vienen deformadas. Tomemos por ejemplo la descripción de 
dos maestras: «La menor de las hermanas tenía una manera muy querida de llevar para todos lados su cuerpo alto [...] Cuando los pies le habían traído el cuerpo cerca de mi silla y ella me obligaba a mirarla levantándome la cabeza con un dedo que enganchaba suavemente en mi barbilla, mis ojos la miraban como a una catedral, y [...] veían muy de cerca el tejido de la tela de su pollera gris en la disimulada montaña de su abdomen» (Hernández, 2008: vol. 3, 13). Así, la elección de un vocabulario junto al uso de metáforas espaciales dan cuenta de un recuerdo visual que aparece ante nosotros totalmente alterado. En Hernández, y con la misma técnica, hasta las impresiones sonoras pueden ser sugeridas por una metáfora espacial, como lo vemos un poco más adelante en el mismo cuento: «Su poca voz tardaba en llegar a la superficie como si tuviera que sacarla de un pozo con una bomba».

Otra de las formas de alterar el espacio mediante la percepción del narrador la tenemos en el relato de una pesadilla. En los sueños, el cerebro crea un universo valiéndose de elementos identificables por el sujeto. El espíritu fabrica un contexto imaginario a partir del mundo en el que evoluciona el personaje y la vuelta al estado de vigilia puede crear un desfase. Así en «Tierras de la Memoria»: «Después de haber dado el grito que hizo derrumbar las paredes del sueño y me dejó con los ojos abiertos en plena noche, seguí revolviendo los escombros para ver de dónde había salido el grito. Al pensar en quién habría sido se me volvió a erizar el pelo» (Hernández, 2008: vol. 3, 72). Aquí se contraponen dos realidades y dos espacios: el sueño y la vuelta a lo real. Y para continuar el juego de cajas chinas, no hay que olvidar al lector que lee este paso y que vuelve a su propia realidad cuando termina el cuento o cierra el libro, este mismo lector que sueña, lo que crea una multiplicación de los pasos hacia otras dimensiones de la realidad.

La inversión es otra técnica pertinente: el espacio puede llegar a ser un personaje, en una dinámica de personificación de los objetos, o, por el contrario, pude operarse una reificación de los personajes, o sea la asociación entre seres vivos y características propia de los objetos. Se trata de borrar las fronteras entre un objeto y un ser animado. Así sucede en el cuento «El balcón», donde el espacio llega a ser el héroe epónimo y actor de la brecha: un balcón, celoso a causa del interés que tiene su propietaria por un joven que viene a su casa para tocar el piano, se derrumba violentamente. Este balcón tiene todas las características de un amante celoso a lo largo del cuento y la ambigüedad se mantiene hasta el final del relato. Otro ejemplo pertinente es la inversión que se desarrolla en el cuento «La pelota»: un niño juega con una pelota fabricada por su abuela a partir de unos trapos. La personificación del objeto altera 
el espacio y contradice las leyes físicas: «Después de haberle dado las más furiosas "patadas" me encontré con que la pelota hacía movimientos por su cuenta: tomaba direcciones e iba a lugares que no eran los que yo imaginaba; tenía un poco de voluntad propia y parecía un animalito; le venían caprichos que me hacían pensar que ella tampoco tendría ganas de que yo jugara con ella» (Hernández, 2008: vol. 1, 115).

Un procedimiento que puede relacionar con la técnica que emplea Silvina Ocampo en su relato «La soga», donde se narra la extraña amistad entre un niño y una cuerda-serpiente. Recíprocamente, la animalización de los hombres aparece en el cuento «Las moscas» de Quiroga, en el cual el personaje humano se transmuta, mediante una estrategia narrativa hábil, en una asquerosa mosca verde, como una anticipación del futuro axolotl cortazariano:

Mas he aquí que esta ansia desesperada de resistir se aplaca y cede el paso a una beata imponderabilidad. No me siento ya un punto fijo en la tierra, arraigado a ella por gravísima tortura. Siento que fluye de mí, como la vida misma, la ligereza del vaho ambiente, la luz del sol, la fecundidad de la hora. [...] Del seno de esta expansión, que el sol dilata desmenuzando mi conciencia en un billón de partículas, puedo alzarme y volar, volar... (Quiroga, 1993: 776).

Por último, la técnica de «mise en abyme» (el relato dentro del relato) altera el espacio en la medida en que borra las fronteras de lo real al crear dos realidades parecidas, una dentro de otra. Esa es la técnica que emplea Hernández en su cuento «Las Hortensias»: el protagonista observa en unas vitrinas a unas muñecas que representan escenas de la vida cotidiana; si pensamos que el lector es un espectador de los actos del protagonista, se opera entonces la «mise en abyme». En esta situación en la que el personaje que observa una escena es también observado, nos recuerda por anticipación la escena del sillón de terciopelo verde de «Continuidad de los parques», de Cortázar. Quiroga, por su parte, va aún más lejos al emplear una «mise en abyme» de lo fantástico, o sea lo fantástico dentro de lo fantástico. Así, en «El síncope blanco», el personaje, desvanecido por efecto del cloroformo que le hicieron respirar en el hospital, crea un universo imaginario en un sueño: un espacio que es la antesala de la muerte y donde esperan los enfermos antes de saber si les llegó la hora o no; allí se encuentra con una paciente de dicho hospital a la que intentará en vano encontrar cuando se despierte. Así, el arte de la escritura estriba en la creación de diferentes «estratos» de realidad para luego confundirlos, pasando de una a otra por atajos vertiginosos. 
Hasta ahora hemos reflexionado sobre elementos textuales recogidos a lo largo de nuestras lecturas. ¿Se podría pensar una geopoética de lo fantástico a otra escala?

\section{3. ¿DÓNDE ESTÁ LO FANTÁSTICO?}

\subsection{El espacio físico, metáfora de lo fantástico}

En este punto de nuestra reflexión, nos parece importante notar que el espacio no tiene solamente un valor referencial o funcional. En efecto, también puede desempeñar un papel simbólico, una función metafórica, lo que no debe sorprender si recordamos la etimología de la palabra «metáfora» y lo que implica en términos de movimiento y de transporte. En el famoso cuento «El almohadón de pluma» de Quiroga, en el cual un asqueroso bicho chupa la sangre de una joven recién casada y va creciendo conforme la mujer pierde toda su energía, la casa blanca tal y como aparece definida al principio del cuento es una anticipación de la palidez progresiva de su habitante, así como en Poe, la fisura de la casa Usher es una prefiguración de la caída y la decadencia de sus habitantes: «La casa en que vivían influía no poco en sus estremecimientos. La blancura del patio silencioso — frisos, columnas y estatuas de mármol - producía una otoñal impresión de palacio encantado. Dentro, el brillo glacial del estuco, sin el más leve rasguño en las altas paredes, afirmaba aquella sensación de desapacible frío», (Quiroga, 1993: 97). Aquí, el espacio constituye un indicio, una clave para dar sentido al cuento en su conjunto, como una metonimia.

Del mismo modo, en el cuento de Felisberto Hernández «La casa inundada», el agua que ha invadido la propiedad y ha transformado los pasillos en ríos, obligando a los personajes a moverse en barco, es el símbolo del fluir de las palabras de la propietaria que cuenta su vida y sus recuerdos al protagonista a lo largo de sus paseos. Así, y para contestar a nuestra pregunta, lo fantástico no estriba solamente en la transformación, la alteración de los espacios, sino también en el valor poético, simbólico de éstos. ¿Cómo superar ahora la dimensión textual del espacio y pensarlo desde la alteridad?

\subsection{Alteridad: los ojos fronterizos}

No hay que olvidar que en el proceso de lectura están los personajes, el autor que escribe y el lector que descubre el texto. Entonces, cuando Roas (2001: 24) afirma «La literatura fantástica es aquella que ofrece una temática tendiente a poner en duda nuestra percepción de lo real», es necesario hacer 
dos preguntas: ¿cómo concebir la noción de percepción? y ¿a quién se refiere el posesivo «nuestra»?

A propósito de la percepción, afirma Merleau-Ponty (2012: 311):

Ce sont surtout les illusions touchant la profondeur qui nous ont habitués à la considérer comme une construction de l'entendement. On peut les provoquer en imposant aux yeux un certain degré de convergence, comme au stéréoscope, ou en présentant au sujet un dessin perspectif. Puisque ici je crois voir la profondeur alors qu'il n'y en a pas, n'estce pas que les signes trompeurs ont été l'occasion d'une hypothèse, et qu'en général la prétendue vision de la distance est toujours une interprétation des signes?

Científica y materialmente, los ojos no son capaces de percibir la perspectiva. Esta es en verdad una construcción de nuestro cerebro. La prueba más evidente de esta idea son las ilusiones ópticas que nos engañan y permiten dos visiones posibles de una sola imagen. En la medida en que el cerebro reconstruye de manera artificial la percepción de la profundidad, ¿sería posible concebir lo fantástico a partir de esta misma idea? La escritura de lo fantástico se relacionaría con una percepción incompleta de los mundos posibles que por eso nos parecen imposibles e inquietantes. Así, por ser aproximativa nuestra percepción de la realidad, y por ser insuficientes los medios lingüísticos de los que disponemos para dar cuenta de ésta, se podría pensar que lo fantástico está en este lugar borroso y en el pasaje del paradigma a nuestra interpretación. La puesta en duda evocada por Roas vendría de la propia condición parcial de nuestra percepción.

A su manera, Felisberto Hernández cuestiona este lugar que son los ojos como un espacio fronterizo entre el exterior y lo interior:

De pronto sentí en el alma un espacio claro, donde vagaba una especie de avión. Voy a suponer que mis ojos miraran para adentro en la misma forma que para afuera; entonces, al ser esféricos y moverse para mirar hacia dentro, también se movían mirando hacia fuera, y por eso yo miraba los objetos que había en mi cuarto, sin atención: yo atendía al avión que andaba adentro y en el espacio claro. Después resultó que la parte de los ojos que miraba para afuera y sin atender, miró — como hubiera podido mirar un enamorado vulgar - una fotografía de ella que estaba en la mesita pintada con nogalina; entonces, en vez de atender al avión de adentro, atendí a la foto de afuera. Después quise volver a ver el avión, pero éste se había perdido en el espacio claro («Las dos historias»; Hernández, 2008: vol. 2, 170-171). 
Con unos puntuales efectos de lo real —la mesita con nogalina en este caso- sobre el telón de fondo general, aparece el movimiento de vaivén entre estos dos espacios.

\subsection{Una nueva perspectiva: descentramiento}

Este concepto surge a partir de los trabajos de los comparatistas que reflexionaron sobre la relación entre espacio físico y literatura. Sin centrarse exclusivamente en juegos intertextuales, una dinámica del geógrafo puede consistir en pensar los lugares en relación con las jerarquías sociales y los espacios condicionados por la mitología, la religión o las ideologías y otros presupuestos que forman el sustrato cultural implícito, el trasfondo del texto o tras-texto.

Si primero nos centramos en la visión de los dos autores que estamos estudiando, no hay que olvidar que, aunque influidos por la literatura y los clásicos europeos, escriben dentro de una realidad, un espacio latinoamericano. Es evidente que la manera de concebir lo fantástico sufre una evolución según el territorio en que nos encontramos. Es lo que llamamos la experiencia del descentramiento cultural e histórico. Si Poe y los autores europeos de su época razonaban a partir de la lógica del mundo exterior sugiriendo lo ilógico por causas que remiten al campo de la razón, Quiroga, por su parte, como otros latinoamericanos, parece pensar a partir del mundo que observa, del espacio que lo rodea. Si en los cuentos europeos y de Poe se trata de subrayar lo que no encaja en nuestra concepción de la lógica, en Quiroga se trata de percibir lo cotidiano con un ojo diferente, desvinculado de la razón e implantado en lo empírico. Por ejemplo, en «Los buques suicidantes», se cuenta una extraña desaparición de los pasajeros de un barco y resulta que el protagonista es el único en sobrevivir y en poder contar su experiencia. El narrador no muere al final porque, en vez de temerla, acepta la maldición que sufre el barco. Así, el hecho de admitir lo fantástico le permite al personaje salvarse la vida. Es cierto que esa situación roza el absurdo kafkiano, en la medida en que el espacio que rodea al personaje contiene tantas aberraciones que no puede ser verosímil. Pero la diferencia que sentimos es que en La metamorfosis Gregor termina asumiendo su estado y hasta se sacrifica para salvar a su familia. En el cuento de Quiroga, se trata justamente de burlar a la muerte para mantenerse en vida y poder contar su experiencia más adelante, y eso mediante la propia aceptación de lo imposible — no absurdo - que rodea al personaje. Esta idea viene confirmada por la visión de Collot (2011): «L'écrivain comme le géogra- 
phe se trouvent confrontés à la nécessité de forger des concepts à la mesure des enjeux d'une description de situations singulières qui permettent de les penser, et, précisément, de traduire la singularité en des représentations communes qui prennent en compte ce rapport dialectique de l'homme au monde qu'il habite et qui l'habite». Se sugiere aquí una aproximación al espacio a través de una dinámica hombre/entorno con una influencia recíproca. Ocurre algo similar en el cuento «Muebles "El Canario"» de Felisberto Hernández: al protagonista, mientras viaja en tranvía, le inyectan algo contra su voluntad y empieza a oír tangos y anuncios de muebles a lo largo del día. No sabemos si esta inyección es lo que provoca las alucinaciones auditivas, pero el narrador lo cree y estos anuncios, que él mismo considera como una propaganda, le contaminan la vida. Aparece aquí una interiorización del mundo exterior que provoca una enajenación, una alienación:

Todavía no había pasado al sueño cuando oí en mí el canto de un pajarito. No tenía la calidad de algo recordado ni del sonido que nos llega de afuera. Era anormal como una enfermedad nueva; pero también había un matiz irónico; como si la enfermedad se sintiera contenta y se hubiera puesto a cantar. Estas sensaciones pasaron rápidamente y en seguida apareció algo más concreto: oí sonar en mi cabeza una voz (Hernández, 2008, vol. 3, 157-158).

Pero otra vez, el protagonista no se resigna y lucha contra su situación insólita. Metafóricamente, se puede leer el cuento como una reacción contra la venidera sociedad de consumo, pero algo interesante aparece al final: el remedio no son las pastillas que necesita vender el hombre de la jeringa, sino un mero baño de pies que, si puede ser visto como una manera común para uno de relajarse, también tiene resonancias bíblicas que superan la anécdota y obligan al lector a convocar otras referencias para comprender lo fantástico del texto.

Por eso, el descentramiento no se aplica solamente a la hora de estudiar cómo y por qué los autores escribieron sus cuentos. Los lectores también pueden experimentarlo con su propio estatuto. Si recordamos el microrrelato «Geografías» de Julio Cortázar, se trata de la visión del mundo por parte de las hormigas dentro de un texto seudocientífico. Unos llamados Horribles Inmensos Seres ${ }^{4}$ serían los hombres, una Gran Sombra Verde, un bosque, entre

4 «Si se va-va-va-va (noción análoga aplicada a la distancia) se llega a la Gran Sombra Verde (¿un campo sembrado, un soto, un bosque?) donde el Gran Dios alza el granero continuo para sus Mejores Obreras. En esta región abundan los Horribles Inmensos Seres (¿hombres?) que destrozan nuestros senderos. Al otro lado de la Gran Sombra Verde empieza el Cielo Duro (¿una montaña?)» (Cortázar, 1994: 461). 
otros ejemplos. Lo que nos importa aquí es notar que toda lectura y percepción son subjetivas. Por eso, también cuenta la lectura libre y personal que cada persona hace del texto. Sin caer en un relativismo yermo, hay que recordar la perspectiva dada por Umberto Eco con el concepto de «obra abierta», según la cual cada lector se apropia el texto no como le da la gana, sino según sus conocimientos y su experiencia de la lectura. El descentramiento estriba en la capacidad del lector de salir de sus certezas y adoptar otro ángulo de vista, como el de las hormigas de Cortázar, para acercarse al texto. Esta podría ser una manera de conciliar las diversas interpretaciones sobre el aspecto fantástico o no de nuestros dos autores. Y esta percepción del lector o del público puede a veces resultar aún más fantástica, como lo sugiere Felisberto Hernández al recordar su infancia: «si cuando era niño me decían que en un teatro daban una obra maravillosa, siempre salía desilusionado, como si antes hubiera esperado que los hombres hicieran volar el techo del teatro y aparecieran desde el cielo cosas más fantásticas que las que salían por los costados del pobre escenario» (Hernández, «Tierras de la memoria»; Hernández, 2008: vol. 3, 23). La imaginación, la loca de la casa, es uno de los actores de lo fantástico de cuyo papel no podemos prescindir, sobre todo cuando el autor nos abre pistas para leer su texto.

\section{CONCLUSIÓN}

Finalmente, queda claro que el espacio tiene diversos avatares: es el espacio presentado en el texto, el espacio vector de transformación del texto fantástico, pero también el espacio fuera del texto, en un juego de interacción entre autor y lector con un cambio de escala.

Claro está que si nos atenemos a la definición de Todorov, poco tienen que ver nuestros autores con esta concepción de lo fantástico. Ahora bien, si los estudiamos mediante el espectro del espacio, con sus matices, con sus técnicas de descripción y de transfiguración, se hace evidente que varios de sus cuentos juegan con la transgresión de lo posible, en un sentido diferente a lo que plantean Caillois o Todorov. En estos casos, la esencia de lo fantástico estriba en un aspecto espacial dinámico: la noción de pasaje de una dimensión a otra de los diferentes mundos posibles e imposibles, en los que la relatividad de la percepción también desempeña un papel. Es un pasaje paulatino, con el que juegan los autores mediante varias modalidades textuales: gramaticales al nivel de la sintaxis de la frase; léxicas cuando trabajan en la semántica y los sentidos de las palabras usadas; teatrales cuando crean una escenificación 
de los mundos descritos que se cruzan o se superponen; simbólicas a la hora de convocar referencias intertextuales cuyos puntos suspensivos completa el lector. Los completa con la imaginación y con la ayuda del autor que, como ya lo decía el propio Quiroga no sin cierta ironía, tiene que guiarle: «Toma a tus personajes de la mano y llévalos firmemente hasta el final, sin ver otra cosa que el camino que les trazaste. No te distraigas viendo tú lo que ellos no pueden o no les importa ver. No abuses del lector. Un cuento es una novela depurada de ripios. Ten esto por una verdad absoluta, aunque no lo sea» («Decálogo del perfecto cuentista»; Quiroga, 1993: 1194).

Así, lo fantástico puede concebirse a través de una doble dinámica: por una parte, se trata de la calidad de la escritura y el uso de los vectores más eficaces posibles para trasponer los elementos de un mundo a otro. La segunda dinámica es la de la recepción, del ojo del lector que comprende el cuento a partir de sus propios conocimientos y siente subjetivamente una brecha con su paradigma. Y terminaremos con una última analogía para explicar nuestra visión de lo fantástico: si pensamos en Hernández, pianista de formación, y en su universo musical, podríamos decir que lo fantástico es quizás lo que hay entre dos teclas de un piano: materialmente, no es nada, es un espacio vacío; pero desde el punto de vista del sonido, entre dos teclas, entre un sonido y su alteración existe lo que los músicos llaman los «comas»; extrañamente, esta misma palabra remite también, desde un punto de vista medical, a un estado de paso, un estado entre la vida y la muerte. Pues entre dos teclas blancas hay nueve comas, lo que implica afinar de cierta manera el teclado para que las teclas negras o alteraciones se sitúen al nivel el cuarto o del quinto coma: es lo que se llama el teclado templado. Cuando está correctamente afinado, las armonías funcionan; cuando ello no ocurre, se produce un desfase, aunque mínimo, una disonancia, que siente el público aficionado a la música. A nuestro parecer allí reside, fugaz pero eficazmente, lo fantástico.

\section{BiBLIOGRAFÍA}

BARON, Christine (2011): «Littérature et géographie: lieux, espaces, paysages et écritures», en Le partage des disciplines, LHT, disponible en < http://www.fabula.org/ lht/8/index.php?id=221> [consultado en enero de 2013].

BARTHES, Roland (1982): «L'effet de réel», en Gérard Genette (ed.), Littérature et réalité, Éditions du Seuil, París, pp. 81-90. 
Borges, Jorge Luis (1989): Obras Completas, Emecé Editores, Buenos Aires.

CAILlois, Roger (2008): CEuvres (ed. Dominique Rabourdin), Gallimard, París.

Collot, Michel (2011): «Pour une géographie littéraire», en Le partage des disciplines, LHT, disponible en <http://www.fabula.org/lht/8/index.php?id=242> [consultado en enero de 2013].

CORTÁZAR, Julio (1994): Cuentos completos, Alfaguara, Madrid.

DURAND, Gilbert (1992): Les structures anthropologiques de l'imaginaire. Introduction à l'archétypologie générale, Dunod, París.

FABRE, Jean (1992): Le miroir de sorcière. Essai sur la littérature fantastique, Librairie José Corti, París.

García, Patricia (2013): «'La frase umbral', cruce al espacio fantástico», en David Roas (ed.), Visiones de lo fantástico. Aproximaciones teóricas, e.d.a, Málaga (en prensa).

HernándeZ, Felisberto (1975): La casa inundada y otros cuentos, Lumen, Barcelona. (1997): Euvres complètes (trad. G. Saad y L. Guille-Bataillon), Seuil, París. (2008): Obras completas, Siglo Veintiuno editores, México, 3 vols.

Merleau-Ponty, Maurice (2012): Phénoménologie de la perception, Gallimard, París.

Muñoz Rengel, Juan Jacinto (ed.) (2009): Perturbaciones. Antología del relato fantástico español actual, Salto de Página, Madrid.

Ocampo, Silvina (1959): La furia y otros cuentos, Sur, Buenos Aires.

Quiroga, Horacio (1993): Todos los cuentos, Archivos, Madrid. (1999): Cuentos, Cátedra, Madrid.

RoAs, David (2001): «La amenaza de lo fantástico», en David Roas (ed.), Teorías de lo fantástico, Arco/Libros, Madrid, pp. 7-44.

(2011): Tras los limites de lo real. Una definición de lo fantástico, Páginas de Espuma, Madrid.

Todorov, Tzvetan (1970): Introduction à la littérature fantastique, Éditions du Seuil, París. WestPhal, Betrand (2007): La géocritique: réel, fiction, espace, Éditions de Minuit, París. 